\title{
CRISPR and The Ethics of Gene Editing: A Modest Framework for Discussion \\ Gerasimos Santas
}

Professor of Philosophy Emeritus, University of California, Irvine, CA 92697, USA

\section{Abstract}

CRISPR is a powerful new biochemical tool that can be used to edit the DNA of somatic cells or the germline. It can be used for good ends such as the treatment or prevention of illness or disability, or for bad ends. All its uses for good ends can be sorted out into six kinds of cases: editing somatic cells to treat or prevent disease-a therapeutic use; editing somatic cells to enhance a living thing; editing the germline to prevent future disease-a second therapeutic use; editing the germline to enhance future living things; editing somatic cells for research to understand nature and unlock its secrets; and editing the germline for the same research purposes. Recent discussions by various experts, in the science, ethics, and governance of gene editing, show confidence in the first kind of case-that it is a good use provided the editing is safe and effective; and hesitation, doubts, and calls to stop editing in the next three cases. The paper explores the basis of our confidence in the somatic therapeutic case, and compares it systematically to the enhancement cases, while using the research cases for what they teach us about good ends. The center of this approach is that our confidence in the therapeutic somatic case is based on our agreement that health is a fundamental good, and the paper suggests an analogy between the ethics of therapy and the ethics of enhancement, which throws some light on the difficult cases of human enhancement.

\section{Introduction}

Jennifer Doudna's and Samuel Sternberg's book, A Crack in Creation [1], is a wonderful introduction to the discovery of CRISPR, a powerful biochemical process for gene editing, to its incredible capabilities, its many uses, and the important questions it raises about the present and future of the plant and animal kingdom and the human race. Doudna and Sternberg (D \& S) tell us that the variety of questions CRISPR raises, bio-technical, ethical, legal and regulatory, cannot be answered only by scientists but need to be considered by the whole society. And indeed the discussions have already included scientists, government, law, and bioethicists. As many have pointed out, the pace of progress in this biotechnology is so rapid that we cannot wait but must discuss these questions now.

A Crack in Creation is written in language accessible to everyone, as a narrative of Doudna's journey to the discovery of CRISPR, conveying both her excitement about this major discovery and her ethical concerns about its uses. A second important book, Human Genome Editing (HGE) [2] is an authoritative and informative report by the National Academy of Science and the National Academy of Medicine, on the Science, Ethics, and Governance of gene editing. Reading these two books was a great learning experience and led me to think about the fundamental ethical issues of gene editing.

As an outsider who has no expertise in the science of gene editing, only some experience teaching ethics, and in the spirit of contributing to public discussion, I make a modest attempt to place the basic ethical questions in a systematic context: is gene editing good or bad, right or wrong, especially in the difficult applications for enhancement of human beings? I have already learned that the ethics of CRISPR can be a complex, difficult, and even an uncomfortable subject, and we can all try for clarity of thought, attention to the facts, to relevant distinctions, and to sound reasoning; all of which, however, have to be accessible to everyone because gene editing concerns everyone.

\section{CRISPR-The Tool and its Basic capabilities}

What is CRISPR and how does it work? What are its present and its future capabilities? These are questions for the scientists to answer, those who discovered and are using gene editing and CRISPR. In A Crack in
Creation (Part I, THE TOOL, Chs. 1-4) Doudna and Strernberg give a very informative and accessible account of the discovery of CRISPR, its main uses so far, and references to their colleagues who are working on gene editing. Human Genome Editing (HGE) also has a very informative discussion of the science and research on gene editing. And Wikipedia, available to everyone free on the Internet, has a long article on CIRSPR with helpful illustrations. CRISPR is a TOOL and as a tool, it has CAPABILITIES. D \& S help us to sort out it main basic capabilities: it can edit the DNA inside cells of living things, plants or animals (whether in a laboratory or in a living thing), by deleting or inserting letters of DNA (the chemical compounds A T, C G); or by changing whole sequences of letters, thus changing the DNA of a gene and the genome of a species. The analogy of editing a text is helpful, but the process of editing DNA is biochemical, while in editing a text there no biochemistry; so the analogy is helpful, but purely formal and rather simplistic. By virtue of these basic capabilities, mutations in genes inside somatic cells (non-heritable material) responsible for any genetic disease in a living thing can be deleted, or inserted, or modified; mutations in genes in the germline (heritable material) can be similarly edited so that future generations are not as susceptible to genetic disease. In D's and S' words: 'As long as the genetic code for a particular trait is known, scientists can use CRISPR to insert, edit, or delete the associated gene in virtually any living plant's or animal's genome." (A Crack in Creation, xiii).

\section{CRISPR-The Tool's Majoruses and their Ethical Dimension}

As a tool CRISPR has many applications, and these uses clearly have an ethical dimension, that is, they can be good or bad, right or wrong. When used to bring about a specific goal, the editing can be beneficial or harmful depending, in part, on the goal for which the editing is

"Corresponding Author: Dr. Gerasimos Santas, Professor of Philosophy Emeritus, University of California, Irvine, CA 92697, USA; E-mail: gxsantas@uci.edu

Citation: Santas G (2019) CRISPR and The Ethics of Gene Editing: A Modest Framework for Discussion. Int J Clin Res Trials 4: 138. doi: https://doi. org/10.15344/2456-8007/2019/138

Copyright: (c) 2019 Santas. This is an open-access article distributed under the terms of the Creative Commons Attribution License, which permits unrestricted use, distribution, and reproduction in any medium, provided the original author and source are credited. 
used. When the editing is designed and used to produce a desirable or good or beneficial goal, then its use is good, provided that the editing is safe (e.g.no off target editing and/or minimal side effects), and effective in reaching the goal; in similarity to earlier gene therapy, drugs, and traditional medical procedures-also tools --, where safety and efficacy are familiar requirements. If the goal is good but there is off target editing or minimal side effects, the editing can still be good on the whole or on balance, provided that the benefits outweigh the harm (favorable risk/benefit analysis). On the other hand, if the editing is designed and used to produce a bad or undesirable or harmful goal, then the editing is also bad, and in this case the more effective it is the worse it is.

\section{Two Distinctions}

When we discuss whether an editing is good or bad or right or wrong, we need to pay careful attention to two distinctions. One distinction is between means and ends: we need to identify means and ends correctly in a particular gene editing use, and be aware of the interplay of means and ends (e.g., in cost/benefit analysis). Confusing means and ends, generally, or in a particular gene editing application, can make it difficult to reach sound conclusions or decisions. In the logic of its capabilities and its means-ends uses, CRISPR is similar to other tools and instruments we are familiar with from our human history-for example, the capabilities and uses of wheels, engines, nuclear energy, solar panels, surgery, drugs, and traditional artificial selection. The uses of all these tools and instruments also have an ethical dimension-their uses too can be good or bad, right or wrong, and we are familiar with ethical discussions of their uses. We can try to judge and evaluate CRISPR's uses in similar ways. Where CRISPR is unusual though not unique, is in its very powerful basic capabilities and the corresponding uses: "The CRISPR/Cas9 system is simpler, faster ... cheaper... highly efficient.... and has revolutionized the field of genome editing...." (HGE, p. 63). A change of one or more letters in the DNA of a gene inside a cell can eliminate or produce a catastrophic illness in a living thing; it can eliminate or produce such an illness in future specimens of a species; and editing the genome can even create a big variation in the future population of a speciesalmost a new species. (D \& Sprovide many examples of current uses of CRISPR, in Chapter 5, The CRISPR Menagerie; many examples are also discussed in HGE, Ch. 4, 5, \& 6).

B \& Sare also helpful in emphasizing again and again the second important distinction, the crucial difference between editing genes of somatic cells and editing the germline. This distinction is also clearly drawn, and forms the division between Ch. 5, Somatic Genome Editing, and Ch. 6, Heritable Genome Editing, in HGE. The editing of genes in somatic cells is not heritable; we are affecting only a single individual. But the editing of the germline is heritable, so germline editing can affect the human genome for generations to come, and the benefits or harms it may cause can be greater, last far longer, maybe non reversible, and are thus more uncertain. Editing of somatic cells can benefit or harm a single specimen; germline editing can affect a whole species, bringing more extensive changes for good or ill.

Using these two distinctions, the applications of gene editing that D\& $S$ discuss-perhaps all applications of gene editing discussed extensively in HGE--can be sorted out into four classes or groups: Somatic therapeutic applications; somatic enhancement; germline therapeutic; and germline enhancement applications. (For these four groups see also HGE, p. 146) [2].

Using the same two distinctions, we have a fifth and sixth important use or two more groups, namely, somatic and germline editing, both for research purposes. These research uses are important because their aim is all important: understanding nature and unlocking its secrets (See B \& S, Ch. 3, Cracking the Code, and HGE, Ch. 3). Without successful research we cannot successfully attempt any clinical applications, the four applications of gene editing, including the urgent and compelling somatic therapeutic. The ethics of research is not the focus of this paper, but these research uses are instructive and revealing and we will return to them.

\section{Somatic Therapeutic Uses of CRISPR}

Editing DNA of somatic cells (whose DNA is not heritable) to treat or prevent a genetic disease or disability in a living thing is usually called a therapeutic use, since it is used to treat disease or restore health-an important good. This is a good or beneficial use of editing, provided that the editing is safe and effective, and has a favorable risk/ benefit analysis (see, e.g. D\& S, Ch. 6, To Heal the Sick). Of course editing of such somatic cells can also be used to produce a disease-and this would be a bad or harmful use-the opposite of a therapeutic use. In therapeutic cases, the ends and means are fairly perspicuous and hard to confuse: the ends are health, or treating or preventing disease or disability; and the editing is the means. Moreover, we already have experience of the somatic therapeutic cases, for example from gene therapy, and most experts think we already have adequate regulations for their safe and effective use. (HGE, Ch. 4, especially pp. 109-10). In these somatic therapeutic cases we see confidence about the means and confidence that the end is good.

\section{Somatic Enhancement Applications}

Here the means are similar to somatic therapeutic applications, the editing of genes in somatic cells, and the regulations already in place seem sufficient for assuring the safety and efficacy of the editing. But the goals are different: instead of the treatment or prevention of disease or disability or the restoration of health, we have the goal of enhancing an individual human being. But what is human enhancement? When we go beyond or outside health (a good to be pursued) and disease (an ill to be avoided), this question seems wide open. This affects the risk/benefits analysis, since what the benefits are is wide open. And when we look at the discussions of this application in the literature we see hesitation and uncertainty about the goal of enhancement, a call for temporary bans and for more discussion. HGE has a subtle and helpful discussion of the distinction between enhancement and therapy; and though there may be borderline cases and intermediates between therapy and enhancement, the distinction is maintained (see HGE, Ch. 6, especially pp, 137-48, and D \& S, Ch. 7 \& 8).

\section{Germline Therapeutic Applications}

CRISPR and other gene editing can also be used, by editing DNA in the germline (heritable material), to prevent genetic disease in future specimens of a species-another good use, another therapeutic use. Of course it can also be used to cause a disease in future specimens of a species-another bad use. Thus this application of gene editing also has a clear ethical dimension-it can be good or bad, beneficial or harmful: and that depends on at least two things: whether its goals are good or bad, and whether the editing (means) is safe and effective. There may be plenty of devils in the details of these uses: e.g. the details of safety, of efficacy, of cost/benefit analysis. Though it may seem essentially similar to somatic therapeutic uses with respect to goals, to treat or prevent disease or disability, editing the germline is far more complicated with respect to means. The effects of the editing of 
specimens, such as viable embryos whose future potential diseases we are trying to prevent, stretch indefinitely into future generations, and there can be more devils in the details. For example, while CRISPR is not error prone it is nevertheless not error proof (See D\& S, 178-81, 214-15, 223-4). Mistakes (e.g. off target editing) may be magnified in future generations; such mistakes may not be reversible; and even the intended effects (the new gene variants) may interact unpredictably with future environments; so we may have far more uncertainty (For the many difficult issues with editing the germline, see also, HGE, Ch. 5 \& 6). Another devil is working out problems with permission and consent for these procedures with embryos: who is to give permission or consent for editing a viable embryo? Current answers are parental autonomy and parental rights (HGE, pp. 120-21); but how far do these rights stretch into the future, to generations of grandchildren and great grandchildren, and on and on? We can presently ask good basic ethical questions about these cases, about the goals and the means to them, in parallel to somatic therapeutic cases. But here, because we are editing the germline, we seem to be stuck with good questions and not confident enough good ethical answers. This use clearly needs a lot of informed and reasoned discussion; and such discussions may need more scientific progress; more accurate editing, for example, before we have reasonable and acceptable answers (See e.g. D \& S, 178-81, for experiments to find more accurate editing). The literature on germline therapeutic editing fully reflects this state of affairs: it is full of caution, uncertainty, calls for temporary bans, and waiting for more scientific progress and more informed discussion. (See HGE, Ch. 5, and final recommendations in Ch. 8, pp 193-4).

\section{Germline Editing to Enhance Human Beings}

Using germline editing to "improve" or "enhance" human beings, in the present and future generations, as distinct from treating or preventing disease or disability (or restoring or maintaining health), is a far more difficult undertaking both scientifically and ethically. This use is similar to the germline therapeutic use in that it involves editing the germline, with all its difficulties; but different in goals since it aims at enhancement or improvement of human beings. And as in the case of somatic enhancement, the goals here can go beyond health and are wide open. Thus this is the most difficult of all applications. We have not only the problems of the means, germline editing, but also the basic problem of what the goal(s) is. What is an enhanced or improved human being?

\section{Enhanced Human Beings}

This basic ethical problem, what is an enhanced human being, is not new, and can be posed historically: in religions, in philosophy, in theology, in poetry, in fiction and the visual arts, there have been many different versions of improved or enhanced human beings. For example, we have images and descriptions of gods who are clearly anthropomorphic-that is, gods as super humans, humans with increased mental and/or physical powers (leaving aside the myth of immortality). And we have many, many versions of these: the several gods of the ancient religions, the gods of the philosophers in ancient times, the gods of the theologians in middle ages, the gods of the modern philosophers and theologians. And in modern fiction and visual arts, we have many versions of "superman" and "wonder woman." Of course, some of these versions of super humans, especially in contemporary science fiction, are electronic versions, which are not be within the powers of gene editing; these are also interesting, technologically and ethically, but we are not concerned with them here, but only with biological versions that can be brought about by gene editing. (For a an informed discussion of present and future electronic versions, see Martin Rees, On the Future Prospects of Humanity, Princeton U.P., 2018, Ch. 3 \&4) [3].

Which of these many versions, if any, or which combination thereof, if any, or what new version of enhanced or improved human beings, do we choose to bring about by CRISPR and other gene editing? How do we evaluate and rank the many versions of enhanced humans so that we can make rational choices- choose the best if we can, or the second best, and so on?

These substantial and fundamental ethical questions are far more difficult to answer than our questions in therapeutic applications.

For one thing, the editing is still the means, but the improved or enhanced version of human being is the end or goal; and that end or goal, an enhanced or improved human being, might not in turn be a means to another goal; so we may not be able to evaluate an enhancement as a means to some further goal. As philosophers and social scientists have argued for a long time, it is a lot easier to evaluate means to an end than the end itself, unless that end is in turn a means to some further goal. And what further ends would we create enhanced human beings for?

The cases where we use gene editing to enhance plants or nonhuman animals are importantly different from the human case. We have already used CRISPR and other gene editing to enhance plants and non-human animals (see D \& S, Ch. 5, for many such uses). In these uses we have in effect treated enhanced plants and animals as means to human ends, such ends being, e.g. better nutrition or health for human beings. And of course in research we have used nonhuman animals for what may be the most important human purpose, to help us understand nature and discover its secrets (See, e.g. D \& S, Ch. 3, Cracking the Code, where this research purpose is displayed at its best).Using such human ends we can decide rationally what enhancements we want in crops or non-human animals. That is how we have solved the problem of what an enhanced plant or enhanced non-human animal is. Some object, of course, to using plants and certainly animals for human purposes, even for research. Perhaps, to put the matter very modestly, it may be only a human conceit, a very old one, that plants and other animals exist for the benefit of human beings and can be used accordingly; not to speak of the fact that many plants and non-human animals existed millions of years before we appeared-they are being used by us for our purposes, but they did not come into being for us.

Using gene editing to enhance human beings is essentially different from using it to enhance plants or other animals, since we have no other beings' purposes as a goal. Creating enhanced human beings for the purposes of other beings, human or non-human, in parallel to creating enhanced plants or non-human animals for the purposes of human beings, would be wrong on the face of it. We need only reflect on the uses of human beings as slaves, as being bred and used for the purposes of other human beings, to see that this would be wrong; and it would not be agreed to even by those who use plants and non-human animals for human purposes without qualms. Our doubts and hesitations about and restrictions to using human subjects in experiments for our research purposes teach us a similar lesson.

Abuses in eugenics also teach us a similar lesson. A eugenics program has three essential parts (and many parts of parts left out here): a conception of what human enhancement is (what the "well" 
is in "well born"), a means to achieving it, and the conditions under which the program is implemented. Plato, for example, who had the first eugenics program for his ideal city in his Repubic, thought that the most intelligent-born (and best educated) humans were the best humans, he proposed artificial selection (modeled after animal selection) to make the best with the best, but used deceptive tactics to mate them (the very opposite of voluntary participation and informed consent). The Nazis notoriously went badly wrong on all three parts: a racist conception of enhancement, massive killings of "lesser races", and violations of every human right. Even in a democratic country like the United States, we had the sterilization abuses that went certainly wrong on voluntary participation and informed consent, to say the least. (In The Gene, An Intimate History, Gale, New York, 2016, S. Mukherjee gives a detailed account of modern eugenics that have gone wrong in one or another of the three essential parts) [4].

If we exclude such objectionable uses of human beings, and we still try to design enhanced humans, what are our ends, and which are good or desirable ends and which not? I try to sketch below a methodical and rational approach for answering this question, without arguing here for any specific results. The center of this approach is to make explicit the assumptions about human good that secure our confidence in the therapeutic applications, and then try to find parallel assumptions about human good for the enhancement uses.

Using our two distinctions, and following D's and S's, and HGE's grouping of various applications where CRISPR or other gene editing might be used, we sorted out all applications of CRISPR and gene editing into four main groups (excluding for the moment the two research uses). These have been arranged from the least difficult, the somatic therapeutic use, to the most difficult, the germline enhancement application; not only in technical difficulties (very important of course but not our focus here), but also difficulty in answering the ethical question of enhancement-the central issue here.

In this ordered series the technical and ethical difficulties are fully noted and reflected in the literature; not only in D \& S but also in the comprehensive HGE, written by a wide and prominent group of leaders in the various subfields. There is consensus that the somatic therapeutic application is the least difficult, that present rules for its use are likely sufficient, and there is confidence in the great benefits of this application of this near miraculous biotechnology (especially in monogenic diseases where a single gene is responsible, and especially where a single letter needs to be edited). At the other end of the series, germline enhancement, we find the very opposite: the consensus here is that the technical difficulties (of, e.g., safety and efficacy) are great, that we need more research to solve these problems, that the risk/benefit analysis is far more uncertain because the benefits are very uncertain, and that we should have a temporary ban on clinical applications, and possibly even on some relevant research. (For this consensus see HGE, Ch. 4, pp. 109-10, and Ch. 8, pp. 192-4, the major conclusions and recommendations of the whole report; D \& S reach similar conclusions).

These two opposite attitudes to these two extreme cases seem to me well informed, reasonable, and prudent. And in any case, I do not write to question them, but rather to explore the differences between the two attitudes, by asking why we have this opposition. The two intermediate cases, somatic enhancement and germline therapeutic, I believe, reveal the two main and very good reasons, one about ends and the other about means. The germline therapeutic case highlights, almost isolates, the difficulties of means, germline editing, since in this case we have no disagreement or uncertainty about the goodness of the ends (treating or preventing disease or disability).On the other hand, somatic enhancement highlights, almost isolates, the difficulty of judging or deciding what human enhancement is, since the somatic editing here is roughly similar to the somatic editing in therapeutic cases. Germline enhancement is the hardest case because it combines these two sorts of difficulties about both means and ends. In this structured context I want to see if we can get some light on an approach to human enhancement.

\section{The Ethics of Therapy and the Ethics of Enhancement}

One way to lay bare the ethics of therapy is to learn from the two therapeutic cases, where we have no doubt about the ends, treating or preventing genetic diseases, present and future, or better health for human beings. In successful somatic therapeutic cases a treated individual is better than s/he was earlier, given some measured baseline before the treatment; and in successful germline therapeutic uses individuals in the future will be better than they are now, given some more complex present baseline for the human population. Whatever other improvements we may want, we want at least lesser disease or better health for the individual or the species; so long as we are confident enough that health is a basic human good and disease something fundamentally bad. The reason why we don't have the usual difficulties about enhancement when we try to make human beings less susceptible to disease or healthier is because we agree, as we should, that health is a fundamental human good, to be pursued as an end, and disease something certainly bad and to be avoided. It is our universal agreement to and confidence that health is a fundamental good and disease a basic ill that gives an ethical basis to and secures our evaluative judgments in the therapeutic uses of gene editing. In these uses we have no doubts about the goals because we have no doubts that health is a great, fundamental good. This might be a bit hidden from us in the literature because the standard way the goal is stated in therapeutic cases is "the treatment or prevention of disease or disability" (the standard use throughout HGE); rather than restoration or maintenance of health; indeed "health" does not even appear in the glossary of this comprehensive work, perhaps because there may be disagreement about its definition. But disease and disability are ills and treating or preventing them is surely an improvement.

Improvement is also fundamental to enhancement. HGE (p. 137) discusses this link, as well as several proposed definitions of enhancement (p. 145).Though none of these definitions offer anywhere near a complete analysis of enhancement, I think because they do not specify any goods as goals, they agree that enhancement is an improvement from an existing condition; and that is something that therapy and enhancement have in common. Of course they are also distinct.

The distinction between the two may not be as hard and rigid as it might appear. See HGE (pp. 137-47), for a sophisticated and helpful discussion; prevention, for example, might be either. D \& S (pp. 23031) also have a helpful discussion of the thin line that sometimes exists between therapy and enhancement, and offer many examples of a "slippery slope" from therapy to enhancement. The slope may be slippery, but dangerous only if we are not sure that the enhancement we end up with is really an improvement.

Therapy and enhancement have in common improvement from an existing condition. They can differ in the goods to be pursued, 
when enhancement aims at goods beyond or outside health. And they usually also differ in the existing condition from which they seek improvement: therapy starts from disease or disability, enhancement from a condition deemed normal or in the normal range. The point here is not to deny the distinction between therapy and enhancement, but to see what they have in common: an improvement from an existing condition, and that improvement in both cases has some important human good as its goal.

Though therapy and enhancement are usually different with respect to goals and baselines, it is important to see that we can have enhancement even with health as the goal, depending on what baseline we start with. If we have an individual with a disease, say, Chronic Lymphocytic Leukemia (CLL), we can measure his/her elevated white blood cell count and use it as a baseline for improvement toward the normal range; treating his/her disease would be standardly regarded as therapy. But suppose his/her white blood cell count is in the normal range (say 4,000 to 10,000 ), but she/he has a known gene variant that makes him/her highly susceptible to CLL, given some trigger; if we can, say, delete that variant and eliminate that risk, we have improved that individual's health over his/her lifetime. And that can count as enhancement-better health over a life time. In both cases we are improving the individual's health, making her healthier or less susceptible to disease. Health can be the goal not only of therapy, as it usually is, but also of enhancement if we start from a base of normal health and aim at greater health. In these respect, we can have similar enhancements with health as the goal in germline editing.

Germline enhancement is more difficult for many reasons, as we have seen. One reason we have not discussed is the difficulty of agreeing on a baseline for the whole human population, from which to judge improvements, whatever the improvements may be. Some have suggested what is "normal" for human beings, others what is "natural" for human beings, others what is "typical". But diseases can occur as naturally as health, often without any human intervention, as different gene variants result from numerous replications of cells. And "normal" (and "typical") as applied to human traits and capacities usually signifies a range or spectrum rather than some privileged or ideal point: e.g. what is normal in height, weight, strength, visual acuity; and many other traits, such as intelligence, which has a huge range among human beings. So where do we start from to improve or enhance or make better human beings, even when we know what traits or conditions we wish to enhance and we know how to do it? Where we start might make a difference whether we are doing therapy or enhancement. It might also make a difference to the natural inequalities that exist among human beings (a "side effect" we should be concerned with). Suppose, for example, that sometime in the future we could by gene editing increase the intelligence of everyone who scores below the normal range on a standard (Stanford-Binet) IQ test, so that no one is below the lowest part of the present range. Would we be doing therapy or enhancement? Well, we would be making human beings smarter and that looks like enhancement if intelligence is a great human good; and we would be decreasing the natural or inborn inequalities in intelligence among humans and leveling (somewhat) the initial playing field of life, thus increasing fairness in life expectations. On the other hand if we used editing only to improve the intelligence of those who score on the retarded range, we might be doing therapy, if being retarded is a disability. Therapy and enhancement in this case have in common making human beings smarter, thus better, at least if we count human intelligence as a great, fundamental good. We can see this commonality at a more abstract level: when successful and satisfy the usual standards of safety, efficacy, informed consent, and favorable risk-benefit analysis, both also satisfy the general principle of beneficence, the principle of promoting well being, supporting providing benefit and preventing harm (HGE, p. 11). Of course this principle and its application presupposes some knowledge or belief about what well being is, and what are benefit and harm.

\section{Health and Other Fundamental Human Goods}

We saw that we are more confident about therapy than about enhancement-something that can be seen clearly in the difference in attitudes between somatic therapeutic and somatic enhancement applications. If our confidence in therapeutic cases rests on our universal agreement that health is a fundamental human good, perhaps we can try to work up enhancements in a parallel way: that is, we can use generally agreed upon fundamental human goods and evils, other than health and disease, to try to specify other enhancements or improvements, beyond health, for present and future humans. For example: physical strength and physical weakness, accurate and erratic memory, higher and lower intelligence, and perhaps several other basic goods and ills, in analogy to health and disease.

Of course some of these enhancements may not be within the scope of CRISPR and other gene editing technologies, if they have no genetic causes; and in such cases CRISPR and other gene editing leaves things alone, perhaps to other technologies or to mother nature. Also, we are here not speaking of socio-economic goods, for example, income and wealth and opportunity and education, which are also not within the scope of gene editing; rather we are speaking about human traits, characteristics, or conditions that gene editing can affect.

Using inventories of agreed upon fundamental human goods and evils, in analogy to health and disease, we could work up a corresponding version of enhanced or improved human beings: for example, not only healthier human beings, but also physically stronger, smarter, with more accurate memories and increased capacities for learning, with greater capacity for knowledge, and so on with other agreed upon and fundamental human goods within the scope of gene editing. Therapy (and enhancement with greater health as a goal), is more comfortable to us and we are more assured about it because we have the great art-science of medicine, now revolutionized by molecular biology and chemistry, with its long experience and safety norms and regulations, and we are confident about the good of health. So we have to be more careful and cautious with enhancements that have other goals, goods other than health. But there are such other fundamental goods; health, however fundamental to human well being, is surely not the only major good.

\section{Two Assumptions}

The approach we have outlined consists in making explicit the assumptions about human good that underlie our confidence in therapeutic cases, and then try to find similar assumptions about human good that can guide enhancement. This approach is itself based on two fundamental assumptions:

First, we assume that in the two enhancement applications of gene editing, what we want to design and bring about is not simply different human being. If simply difference is the goal, only our imaginations are the limit. This is not to downgrade human imagination in any way; it is likely responsible for some of our greatest achievement in poetry, fiction, the visual arts, music, and perhaps science. (D \& S appreciate imagination too, when at the beginning of their book (p. viii) they quote Emerson, "Science does not know its debt to imagination"). 
Of course any human beings we design for enhancement by gene editing would be different from what we are now, and in this imagination may indeed have a big role. But here we are talking about designing improved or enhanced human beings-making BETTER human beings (or making human beings better at, e.g., interactions with their environments). Among the many different versions of human beings we can imagine, some of which we know historically, and some of which we can bring about by editing, we want to know which would be better and which worse. And if that is so, we must rely on what we confidently think, and generally agree on, is fundamentally good and bad for human beings; at least among attributes of human beings or personal characteristics within the scope of gene editing, as distinct from social and environmental goods and evils. Our task here is evaluative to begin with, though not only evaluative-to decide what are better human beings and what makes them better-and there is no way we can do that and design better human beings without the assumption of fundamental human goods and ills.

There is an understandable tendency or desire to try to avoid evaluations (value judgments) as much as possible (see, e.g. HGE, ch. 6 especially pp. 137-140). There are legitimate concerns, as we saw, with using the value laden terms "normal" and "natural" (even "mutation" is replaced by "gene variant"). More substantially, in the context of evolution we have learned that what is or confers an advantage in one environment may not be so in another (the example of the sickle cell disease is sometimes cited-the gene variant responsible for it can confer an advantage in malaria infested environments).But it is by no means clear that this relativity of advantage to environment extends to all advantages or all human goods. After all, even these variable advantages are judged to be advantages or not, given the assumption that human survival and reproduction are fundamentally good things.

This tendency or desire to avoid value judgments is understandable, because it is difficult to secure objectivity in evaluations, and certainly difficult or even impossible to experiment in order to confirm or disconfirm value judgments, especially about final ends.

Nevertheless, it may also be difficult, even impossible, to avoid value judgments about goals and ends, even in scientific work. The research uses of gene editing, for example, assume that understanding nature and unlocking its secrets is a great human good, indeed a noble end to pursue and give one's lifetime work to, sometimes at high cost. We don't argue whether understanding nature is a great good, but only about how to design and do research that helps us reach that end. And all the four major applications of gene editing we have discussed which have good goals assume that safety and efficacy are also great goods, this time pertaining to means. No one argues whether safety or efficacy in gene editing are great goods to be pursued, but only how to secure them, even when we are not certain about the ends they are to serve (as in the enhancement cases).

Second, we assume that while there may be disagreements about some human goods and their order of importance (priorities among them), there can also be some general consensus on fundamental human goods. The choice of human health and human disease as evident examples of fundamental and generally agreed upon human good and bad, so prominent and assumed in therapeutic uses, and so fundamental to medicine is no accident. The presence of health and the absence of disease are so fundamentally good because health is a necessary condition for so many human activities and the enjoyment of so many other goods, while severe disease is catastrophic for nearly everything. And we do have universal agreement that health is good and disease an ill: we do not dispute whether health is a good (or disease something bad), even when we are not sure about their definitions; we dispute rather about sources and means to health, access and availability and distribution of such means as nutrition and medical care and health insurance.

Accurate human memory, a necessary condition for learning-the great human capacity for learning being a fundamental human good-, may be another example of a similarly fundamental good; intelligence perhaps another. Can we agree on some such fundamental goods and evils, as we do for health and disease? If we can, after reflections and discussion, then we have a chance to design future humans with some confidence that they really are better humans.

We should remember too that perhaps we are overdoing out doubts and concerns about enhancement, especially in the germline editing case where we have great difficulties about the editing itself (its safety and efficacy). Past misuses and abuses in eugenics, for example, are often cited as a basis for doubts and hesitations about enhancements. And some doubts and hesitations are certainly in order. But we must also remember that judgments of such misuses or abuses presuppose some confident judgments about good and evil-how else would we know these are misuses or abuses? Perhaps some other basic goods, besides health, are equally evident and really generally agreed on. I was startled when a colleague, with whom I was discussing these issues, turned to me and said, "What parents would not want their children to be stronger and smarter?" Greater physical strength and greater smarts may be goods as fundamental as health. S \& D themselves have no problem reeling off two kinds of enhancements "that come to mind... high intelligence, prodigious musical ability, mathematical prowess, tall stature, athletic skills, or stunning beauty," though, they point out, these are too complex for CRISPR, at least for now. Other enhancements within CRISPR's powers are "exceptional levels of endurance... extra strong bones... leaner muscles... lower requirements of daily sleep." (D \& S, p. 230). While some of these might be disputed, and universal distribution of the enhancements in the first set might be very problematic (do we want everyone to be Mozart?), there seem to be many other human goods besides health for enhancements to aim at.

Of course often therapy has priority over enhancement because disease can be so disabling. But putting our health at the top or near the top of our priorities among major human goods does not thereby knock others off the list of such human goods. And some might count human intelligence, for example, above even health, generally, even though in special medical situations health might still be first to try to improve or maintain. Further, human intelligence might be something good to have in any environment, as presumably we judge health to be.

\section{Other Problems about Gene Editing}

Finally, even if we give this approach about enhancement the benefit of every doubt, we still have other problems about gene editing, technical, ethical, and practical. It is important not to conflate them, even if several are interdependent.

We have already mentioned biotechnical challenges about safetyhow to make editing more accurate. And we have ongoing experiments about how to target what we wish to edit without triggering editing of other partially similar sequences. We have also technical issues about efficacy. 
And we mentioned ethical challenges about permission or consent, or autonomy, concepts rather difficult to apply directly to embryos.

We also noted the important ethical problem about the distribution of the benefits we can bring about by CRISPR among human beings. Here we have questions of justice and fairness that must be raised about all four main applications of gene editing. In cases of therapy, who is to benefit from gene editing? For example, all human beings absolutely equally? Or in proportion to their health or disease or disability? Or in proportion to some other needs, such as understanding nature and our place in it? In cases of enhancement, even assuming we know what enhancement is, should enhancement be regarded as a kind of luxury, compared to therapy, and be left wide open to individual tastes and resources? At least somatic enhancement might be left to individual choice as it is now with some, e.g., cosmetic enhancements; while heritable enhancement clearly concerns all human beings and all should have a voice in it.

We also have issues of practical application of gene editing, similar to availability and access to vaccinations, given the large numbers of human beings.

And we have problems of compliance with any agreed upon laws and regulations about gene editing; even if we can reach global agreements on laws and regulations, some might disobey and try to create human beings to serve their own private ends and ambitions. This concern is often expressed by S \& D and it is of major importance. It is difficult if not impossible to have one hundred percent compliance in any law or norm; and when it comes to global compliance to global agreementsthe present case of gene editing-the difficulties can be formidable even to contemplate.

All these issues are important and urgent and need more research and discussion and hopefully general consensus; but they are not the center of this paper. Here we have focused mainly on suggesting a methodical approach to the main ethical question, whether gene editing is good or bad, right or wrong, especially in the difficult cases of editing for enhancement, hoping to understand better what enhancing or improving a human being would be. Given the extremely rapid rate of development of the biotechnology of gene editing, the question of what human enhancement is seems urgent indeed. A temporary ban on editing for enhancement is not only prudent, but a valuable, maybe unique opportunity for fruitful discussion. If we have the capacity to create different human beings, we better try hard to find out which, if any, among many imagined different human beings would be better than what we are now. This concerns every human being and everyone should have a voice in it.

\section{Competing Interests}

The author declare that there is no competing interests regarding the publication of this article.

\section{Referenes}

1. Doudna J, Sternberg SH (2017) A Crack in Creation, Gene Editing and the Unthinkable Power to Control Evolution. Houghton Mifflin Harcourt, New York, 2017.

2. Human Genome Editing, Science, Ethics, and Governance (2017) The National Academy of Sciences, The National Academies Press, Washington DC

3. Martin R (2018) On the Future Prospects For Humanity, Princeton University Press, Princeton \& Oxford.

4. Mukherjee S (2016) The Gene, An Intimate History, Gale, New York. 